\title{
TEMAS AGRARIOS \\ Evaluación de diferentes sustratos enriquecidos con microorganismos para la producción de compost en áreas naturales
}

\section{Evaluation of different substrates enriched with microorganisms for the compost production in natural areas}

\author{
Lázaro J. Ojeda-Quintana ${ }^{1 * \oplus}$; Consuelo Hernández-Rodríguez ${ }^{2}$ \\ Anaisa López-Melian ${ }^{3}$; Celso Frómeta-Milanés ${ }^{1 \odot}$ \\ Recibido para publicación: agosto 18 de 2020 - Aceptado para publicación: diciembre 27 de 2020
}

RESUMEN

La práctica de elaboración y aplicación del compost se acepta ampliamente como sostenible en la producción de cultivos. La investigación se realizó en la Unidad Científico Técnica Base "UCTB Suelos Cienfuegos", Cuba con el objeto de evaluar el proceso de compostaje a partir de diferentes combinaciones de residuos de plantas (Sacharum officinarum, Pennisetum purpureum, Leucaena leucocephala, Eichhornia azurea y Oryza sativa) y la adición de un inoculante microbiano. Se condujeron cinco tratamientos con tres repeticiones en un diseño de Bloques al azar. Se midió la temperatura durante todo el proceso, la descomposición y se determinó la composición química del compost obtenido. El comportamiento de la temperatura mantuvo indistintamente en todos los tratamientos la secuencia de fases: Mesófila, Termófila y Mesófila II, con una duración del proceso de compostaje en los tratamientos que contenían $S$. officinarum y $P$. purpurea entre 105-110 días y un porcentaje de descomposición de $78,2 \%, \quad 84,6 \%$ y $88,6 \%$ respectivamente; mientras que, en los tratamientos que contenían $E$. azurea y $O$. sativa la duración estuvo entre 135-140 días, con una descomposición de 68\% y $69,4 \%$ respectivamente, sin que dicha extensión influyera en la descomposición alcanzada. Al evaluar los resultados se concluye que los tipos de compost obtenidos son viables para su uso, teniendo en cuenta sus propiedades, con destaque para el tratamiento con $S$. officinarum 3 , que tuvo los mayores valores de nitrógeno $1,55 \%$, potasio $1,87 \%$, materia orgánica $62,8 \%$, fósforo de 0,53\%, relación carbono nitrógeno 23,29 y el mayor porcentaje de descomposición.

Palabras clave: Descomposición; Micoroorganismos; Residuos vegetales; Temperatura.

${ }^{1}$ Centro Universitario Municipal Cumanayagua, Universidad de Cienfuegos, Cuba.

${ }^{2}$ UCTB Suelos Cienfuegos, Ministerio de la Agricultura, Cuba.

${ }^{3}$ Facultad de ciencias Agrarias, Universidad de Cienfuegos, Cuba

*Autor para correspondencia: Ph.D. Lázaro Ojeda Quintana

Email:joberoverde@azurina.cult.cu

\begin{abstract}
The fabrication and use of compost are accepted thoroughly as a sustainable practice. In the "UCTB Soils Cienfuegos", Cuba, a research was carried out with the objective of evaluating the composting process starting from different combinations of plant residues with the addition of a microbial inoculant. A completerandomized block design with five treatments, and three replicates was used. The temperature was measured during the whole process, the decomposition and the chemical composition of the obtained compost was determined. Temperature maintained, indistinctly, for all treatments the sequence of the phases: Mesophyll, Thermophile and Mesophyll II, with a duration of the composting process between 105-110 days, and a percentage of decomposition of $78,2 \%, 84,6 \%$ and $88,6 \%$ respectively in the treatments 1,2 and 3 . In treatments 4 and 5 , the duration of the process extended between 135-140 days, with a decomposition of $68 \%$ and $69,4 \%$ respectively, without effect on the decomposition level. The results allow to conclude that all compost types obtained are viable for use provided based on compost properties. Treatment 3 showed the lergest nitrogen value $(1,55 \%)$, potassium $(1,87 \%)$, organic matter $(62,8 \%)$, phosphorus content of $0,53 \%$, carbon/nitrogen $(\mathrm{C} / \mathrm{N})$ rate 23,29 and the highest percentage of decomposition.
\end{abstract}

Keywords: Decompositions; Microorganisms; Vegetable decays; Temperature.

Cómo citar

Ojeda-Quintana, L.J., Hernández-Rodríguez, C., LópezMelian, A. y Frómeta-Milanés, C. 2020. Evaluación de diferentes sustratos enriquecidos con microorganismos para la producción de compost en áreas naturales. Temas Agrarios 25(2):129-140 https://doi.org/10.21897/rta.v25i2.2455

Temas Agrarios 2020. Este artículo se distribuye bajo los términos de la Licencia Creative Commons Attrubution 4.0 (https://creativecommons.org/licenses/ by-nc/4.0/deed.es), que permite copiar, redistribuir, remezclar, transformar y crear a partir del material, de forma no comercial, dando crédito y licencia de forma adecuada a los autores de la obra. 


\section{INTRODUCCIÓN}

El compostaje proporciona la posibilidad de transformar de una manera segura los residuos agrícolas en insumos productivos y contribuye a la reducción de emisiónes de gases de efecto invernadero. Aunque la gran mayoría de los materiales orgánicos son compostables, se deben tener en cuenta los parámetros que afectan su crecimiento y producción. Estos factores incluyen el oxígeno o aireación, la humedad del sustrato, temperatura, $\mathrm{pH}$ y la relación carbono nitrógeno $(\mathrm{C} / \mathrm{N})$ (Román, et al., 2013).

Una de las estrategias para la mitigación del cambio climático es la captura de carbono orgánico en el suelo (COS), principalmente en sistemas de uso de la tierra (Alvarado et al., 2013), donde el compostaje resulta una práctica de gran utilidad, al facilitar la transformación de los residuos orgánicos (por demás uno de los problemas ambientales derivado de la agricultura) en un producto nutricional para aplicar al suelo como enmiendas o abonos.

La composición del compost varía de acuerdo a la duración del proceso de elaboración, actividad biológica y tipos de materiales que se utilicen. Su calidad se determina a través de propiedades físicas, agroquímicas, biológicas, y del contenido nutricional para proveer nutrientes a un cultivo (García et al., 2014).

Raras veces un material tiene todas las condiciones físicas y químicas adecuadas, es por eso que en la mayoría de los casos es necesario mezclarlo con otros materiales. Por lo cual es muy importante conocer las propiedades de cada componente y analizar la incidencia de todas las variables internas y externas que intervienen en el proceso para garantizar un producto de alta calidad nutricional (Carlile et al., 2015).
En Cuba, a partir de la década de los noventa, se incrementó el empleo de residuos orgánicos para la producción agrícola, incluida la práctica del compost (Vento et al., 2010), por su parte Casimiro, (2016), refiere que en el sistema alimentario cubano, dependiente en alto grado de las importaciones, se necesitan transformaciones en su modelo de desarrollo agropecuario; que se basen fundamentalmente en una producción más endógena y en el uso más eficiente de los recursos localmente disponibles de una manera ecológicamente sostenible, lo cual convierte al compostaje en una alternativa sustentable.

En la actualidad cubana, la tecnología de elaboración de compost se practica en mayor o menor medida; no obstante, el volumen de producción no resulta suficiente para satisfacer las demandas agrícolas y además es necesario perfeccionar los métodos de elaboración para que el mismo sea extendido a las grandes empresas estatales y al sector privado que requiere de estos insumos para garantizar la sostenibilidad de sus producciones.

Tomando en cuenta los beneficios, que desde el punto de vista agrícola y medioambiental trae consigo el compost, el presente trabajo tiene como objetivo evaluar las principales variables que intervienen en la producción artesanal de compostaje, a partir de diferentes combinaciones de residuos de plantas y la adición de un inoculante microbiano para acelerar el proceso, así como, determinar la composición química del compost obtenido.

\section{MATERIALES Y MÉTODOS}

\section{Ubicación del área de estudio.}

El experimento se condujo en un área bajo sombra de la Unidad Científico Técnica de Base "Suelos Cienfuegos", ubicada en las coordenadas $22^{\circ} 09^{\prime}$ de latitud norte y $80^{\circ}$ $12^{\prime}$ de longitud oeste, a $60 \mathrm{msnm}$, poblado 
de Barajagua, municipio de Cumanayagua, provincia de Cienfuegos, región centro sur de Cuba, perteneciente al Ministerio de la Agricultura.

\section{Diseño y tratamiento experimental.}

Se utilizó un diseño experimental en Bloques completos al azar con cinco tratamientos y tres repeticiones, para un total de 15 unidades experimentales: Tratamiento 1: Caña de azúcar (Saccharum officinarum L.) + Estiércol vacuno + Inoculante; Tratamiento 2: King grass (Pennisetum purpureum Schumach vc: Común) + Estiércol vacuno $+5 \%$ de Leucaena leucocephala L. vc: Perú + Inoculante; Tratamiento 3: Caña de azúcar (Saccharum officinarum L.) + Estiércol vacuno $+5 \%$ de Leucaena leucocephala L. vc: Perú + Inoculante; Tratamiento 4: Jacinto de agua (Eichhornia azurea K.S) + Estiércol vacuno + 5\% de Leucaena leucocephala L. vc: Perú + Inoculante; Tratamiento 5: Paja de arroz (Oryza sativa L.) + Estiércol vacuno + Jacinto de agua (Eichhornia azurea K.S) + Inoculante.

\section{Procedimiento experimental}

El Inoculante consistió en una mezcla de los microorganismos Aspergillus oryza, Bacillus nato y Sacharomyces cerevisiae obtenidos en Laboratorio de micorobiología y mezclados para su aplicación con humus de lombriz, a razón de 4 kg/t peso verde (Mayea, 1992). El estiércol vacuno utilizado estaba descompuesto.

Las pilas o montones dinámicos (windrow) se confeccionaron al aire libre, sobre un suelo Pardo Grisáseo (Hernández et al., 2015), en forma de meseta suave, con dimensiones de $5 \mathrm{~m} \times 2 \mathrm{~m} \times 1.5 \mathrm{~m}$, para un total de $15 \mathrm{~m}^{3}$, conformadas en capas en el orden siguiente: primera capa de $20 \mathrm{~cm}$ con las combinaciones del sustrato vegetal, segunda con Inoculante en espolvoreo y tercera $10 \mathrm{~cm}$ de estiércol vacuno descompuesto, en la variante correspondiente. Este proceso se repitió hasta lograr la altura concebida.

Los residuos de caña, king grass, paja de arroz y jacinto de agua fueron troceados en fragmentos de $10 \mathrm{~cm}$ aproximadamente y pesados con una balanza digital en el momento de conformar cada capa para garantizar una estructura y granulometría uniforme. La Leucaena troceada se adicionó por capas en un 5\% en relación al peso total de los sustratos. Durante el proceso de compostaje a las pilas se le aplicó riego manual con agua para mantener humedad. Se tuvo en cuenta la lluvia precipitada. Decenalmente se voltearon las pilas.

\section{Mediciones realizadas.}

La temperatura se midió diariamente en cuatro puntos interiores de las pilas con un termómetro de suelo, a una profundidad entre $25-30 \mathrm{~cm}$. Cuando la temperatura en el interior de las pilas fue inferior o igual a la ambiental concluyeron todas las mediciones y se consideró ese momento como el inicio de la fase de maduración. Se dejaron inmóviles hasta que la apreciación visual identificara en el compost un color similar a "borra del café". (Mayea, 1992).

\section{Caracterización de los componentes y el compost.}

Para caracterizar los componentes iniciales y las propiedades del compost obtenido se tomaron un total de 15 muestras de 350 gramos por cada uno de los tratamientos y se determinaron los indicadores que se aprecian en la Tabla 1. 
Ojeda-Quintana. et al. - Sustratos para la producción de compost

Tabla 1. Métodos empleados en los análisis de laboratorio

\begin{tabular}{lll}
\hline Variables & \multicolumn{1}{c}{ Método } & \multicolumn{1}{c}{ Equipo } \\
\hline $\mathrm{pH}\left(\mathrm{H}_{2} \mathrm{O}\right)$ & Electrométrico & $\mathrm{pH}$-metro \\
C-orgánico & Calcinación & Estufa \\
Nitrógeno (NRAG 564, 2003) & Colorimetría & Espectrómetro \\
Fósforo (NRAG 564, 2003) & Colorimetría & Espectrómetro \\
Potasio (NRAG 564, 2003) & Colorimetria & Espectrómetro \\
Relación C/N (NC-10390,1999a) & Cálculos & - \\
Materia orgánica (NC-51,1999b) & Cálculos & - \\
\hline
\end{tabular}

El porcentaje de descomposición de las pilas fue calculado de la forma siguiente:

$$
\% \text { Descomposición }=\frac{\text { Peso seco inicial }- \text { Peso seco final }}{\text { Peso seco final }} \times 100 \text {, dónde }
$$

Peso inicial y Peso final $=\frac{\text { Peso verde inicial } x \text { Materia seca total inicial } y \text { final }}{x 100}$

\section{Análisis estadísticos}

Se realizó una prueba de normalidad de los datos, luego se llevó a cabo un análisis de varianza con cada una de las variables de respuesta, con el fin de establecer las diferencias entre tratamientos. Posteriormente, se efectuó una prueba de Tukey al 5\%. Los datos se procesaron con el paquete estadístico Stadistical Package for Social Sciences (SPSS) para Microsoft Windows ${ }^{\circledR}$, versión 15.0.

\section{RESULTADOS Y DISCUSIÓN}

\section{Caracterización de los componentes}

Entre los parámetros de seguimiento para la obtención de compost se encuentran la temperatura, la humedad y el pH. Relativo a la naturaleza del sustrato lo caracteriza el tamaño de partícula, la relación carbono-nitrógeno
$(\mathrm{C} / \mathrm{N})$, los nutrientes, la materia orgánica y la conductividad eléctrica (Donn et al., 2014).

La Tabla 2 muestra el contenido de nitrógeno, fósforo, potasio, materia orgánica y la relación $\mathrm{C} / \mathrm{N}$ de los materiales utilizados en la elaboración del compost. Se aprecia el mayor porcentaje de nitrógeno en la Leucaena, especie de leguminosa utilizada comúnmente por los productores como suplemento proteico en la alimentación animal (3,23\%), con diferencias del resto de los tratamientos, seguido del estiércol vacuno $(1,61 \%)$, que no difirió del Jacinto de agua (1,30\%), y este a su vez sin diferencias con la paja de arroz $(1,20 \%)$. Los valores más bajo de nitrógeno, y sin diferencias entre sí, estuvieron en la caña de azúcar y el king grass, con 0,79\% y 0,80\% respectivamente. 
Ojeda-Quintana. et al. - Sustratos para la producción de compost

Tabla 2. Composición química de los residuos vegetales utilizados en el compostaje

\begin{tabular}{lccccc}
\hline \multicolumn{1}{c}{ Residuos } & $\mathbf{N}(\%)$ & $\mathbf{P}(\%)$ & $\mathbf{K}(\%)$ & $\mathbf{M . O}(\%)$ & $\mathbf{C} / \mathbf{N}$ \\
\hline Caña de azúcar & $0,79^{\mathrm{d}}$ & $0,24^{\mathrm{c}}$ & $0,34^{\mathrm{d}}$ & $89,13^{\mathrm{a}}$ & $64,84^{\mathrm{a}}$ \\
King grass & $0,80^{\mathrm{d}}$ & $0,27^{\mathrm{c}}$ & $0,31^{\mathrm{d}}$ & $86,62^{\mathrm{a}}$ & $62,23^{\mathrm{a}}$ \\
Leucaena & $3,23^{\mathrm{a}}$ & $0,68^{\mathrm{b}}$ & $2,55^{\mathrm{c}}$ & $91,41^{\mathrm{a}}$ & $16,26^{\mathrm{c}}$ \\
Jacinto de agua & $1,30 \mathrm{~b}^{\mathrm{c}}$ & $0,92^{\mathrm{ab}}$ & $7,23^{\mathrm{a}}$ & $34,75^{\mathrm{c}}$ & $15,51^{\mathrm{c}}$ \\
Paja de arroz & $1,20^{\mathrm{c}}$ & $0,33^{\mathrm{c}}$ & $3,47^{\mathrm{b}}$ & $67,10^{\mathrm{b}}$ & $32,14^{\mathrm{b}}$ \\
Estiércol vacuno & $1,61^{\mathrm{b}}$ & $1,08^{\mathrm{a}}$ & $3,20^{\mathrm{b}}$ & $87,12^{\mathrm{a}}$ & $31,10^{\mathrm{b}}$ \\
\multicolumn{1}{r}{ es \pm} & $0,0784^{*}$ & $0,050^{*}$ & $0,144^{*}$ & $1,601^{*}$ & $2,124^{*}$ \\
\hline
\end{tabular}

Letras distintas indican diferencias significativas según la prueba de Tukey $(p \leq 0,05)$.

En cuanto al fósforo, el estiércol vacuno fue superior $(1,08 \%)$, sin diferir del Jacinto de agua $(0,92 \%)$, que no marcó diferencias estadísticas con la Leucaena $(0,68 \%)$. El resto de los tratamientos no difirieron entre sí con $0,33 \%$ la paja de arroz, $0,27 \%$ el king grass y $0,24 \%$ la caña de azúcar El potasio superó estadísticamente a todos los tratamientos en el Jacinto de agua $(7,23 \%)$, seguido de la paja de arroz $(3,47 \%)$, el estiércol vacuno $(3,20 \%)$, sin diferencias entre sí, y la Leucaena $(2,55 \%)$. El contenido más bajo de este elemento, y sin diferencias estuvo en la caña de azúcar y el king grass con $0,34 \%$ y $0,31 \%$ respectivamente.

En cuanto al contenido de materia orgánica los residuos de Leucaena $(91,41 \%)$, caña de azúcar $(89,13 \%)$, estiércol vacuno $(87,12 \%)$ y king grass $(86,62 \%)$ no difirieron entre sí, con los valores más elevados de este indicador. Los residuos de paja de arroz $(67,10 \%)$ y Jacinto de agua $(34,75 \%)$ fueron los más bajos en materia orgánica con diferencias entre sí.

El comportamiento de la relación $\mathrm{C} / \mathrm{N}$ mostró el valor más alto en la caña de azúcar $(64,84)$ y el king grass $(62,23)$, sin diferencias estadísticas entre ellos, pero sí del resto de los tratamientos, seguida de la paja de arroz $(32,14)$ y el estiércol vacuno $(31,10)$, sin diferencias. El Jacinto de agua $(15,51)$ y la Leucaena $(16,26)$ fueron los valores más bajo de la relación carbono nitrógeno en los residuos vegetales utilizados como materia prima para el compostaje.

Señalar que los sustratos caña de azúcar y king grass no mostraron una relación $\mathrm{C} / \mathrm{N}$ cercana o igual al rango 25-35/1, donde los microorganismos inician el proceso de degradación en las pilas con mayor efectividad, incluso, pudieran ralentizar el mismo en presencia de esta cantidad de carbono (Rodríguez et al. 2018), por su parte, Escudero y Arias, (2012) destacan la influencia que ejerce la relación $\mathrm{C} / \mathrm{N}$ inicial sobre el proceso de compostaje y los microorganismos (a menor relación pero cercana al 35/1 teórico, menor duración del compostaje y microbiota más abundante).

El material de partida y las condiciones del hábitat a su vez, inciden en la composta y en el tipo de microorganismos que gobiernan el abono que se obtenga. De ahí la necesidad de añadir material rico en nitrógeno en los casos donde la relación $\mathrm{C} / \mathrm{N}$ no resulte favorable, ventaja que ofrece combinar los diferentes componentes empleados como materia prima. En el proceso de compostaje el carbono es la fuente de energía utilizada por los microorganismos para la activación de sus procesos metabólicos, mientras que el 
nitrógeno, es el material básico para la síntesis de material celular, por lo tanto la relación $\mathrm{C} / \mathrm{N}$ es uno de los aspectos más importantes en el balance nutricional del compost.

La influencia que ejerce la relación $\mathrm{C} / \mathrm{N}$ en el proceso de compostaje ha sido señalada por Bohórquez et al., (2014), al evaluar la calidad de un compost elaborado con diferentes combinaciones de subproductos del proceso de molienda de la caña de azúcar (Saccharum officinarum L.) en el Ingenio Riopaila-Castilla, Valle del Cauca, Colombia, al determinar que la relación carbono-nitrógeno inicial de las mezclas es fundamental para obtener una buena calidad del compost y puede condicionar el tiempo de compostaje para una adecuada maduración con máximo contenido de nutrientes a los 90 días.

\section{Dinámica de la temperatura en el proceso de compostaje}

En la Figura 1 se aprecia el comportamiento de la temperatura en el interior de las pilas durante el proceso de compostaje. Se observó que en los primeros 20 días estuvo marcada por una fase Mesófila $\left(35,8-42,4^{\circ} \mathrm{C}\right)$, donde las especies de microorganismo mesófilos tienen un intervalo de temperatura óptima en el que su actividad es mayor y más efectiva. En la tercera y 4ta decena, solo los tratamientos 1 y 3 alcanzaron la etapa Termófila $\left(45-70^{\circ} \mathrm{C}\right)$, aunque la temperatura no superó $\operatorname{los} 51^{\circ} \mathrm{C}$, fase donde un grupo de microorganismos termófilos intervienen y ocurre un proceso de saneamiento. En la 5ta decena se reportó el retorno a la fase Mesófila II, ya en la 6ta y $7 \mathrm{ma}$ disminuyeron las temperaturas e igualaron la temperatura ambiental.

El tratamiento 2 continuó una tercera decena todavía en fase Mesófila, y seguidamente comenzó un proceso paulatino de descenso de la temperatura. En los tratamientos 4 y 5 desde la tercera decena la temperatura estuvo en valores iguales e inferiores a la temperatura ambiental, sin transitar a la fase Termófila.

Durante el compostaje, la temperatura se mueve desde valores ambientales hasta la más adecuada para los microorganismos mesófilos (cerca de $40^{\circ} \mathrm{C}$ ), luego intervienen los microorganismos termófilos, entre $40^{\circ} \mathrm{C}$ y $75^{\circ} \mathrm{C}$, seguido de una etapa Mesófila II que transita a un enfriamiento hasta la temperatura ambiente, que indica la maduración (INN 2004), citado por Escudero y Arias, (2012).

Evidencias del comportamiento de la temperatura durante la elaboración de compost, reportan López et al., (2017), en un compost elaborado a partir de bagazo, cachaza y ceniza, obtenidos del proceso de fabricación de azúcar con 0,2 \% de urea como aditivo activador, donde hubo en los primeros 25 días un incremento lineal en la temperatura hasta alcanzar valores promedios de $55^{\circ} \mathrm{C}$. A partir de los días 25 y hasta los 35, la temperatura se mantuvo estable y posteriormente los valores descendieron de forma gradual. En el presente trabajo la temperatura más alta se alcanzó entre los 30 y 40 días con mayor permanencia de la misma que la reportada por estos autores.

En el compostaje se debe tomar en cuenta la influencia del tamaño de los materiales, por lo tanto la aireación en las pilas o la retención de humedad, pudieran estar estrechamente relacionadas con el tamaño de la partícula, y a su vez incidir en la temperatura durante todo el proceso. Trocear uniforme los componentes resultó favorable para la elaboración del compost.

Autores como Campos et al., (2016), evaluaron técnicamente dos métodos de compostaje de residuos sólidos biodegradables con miras a utilizar el abono resultante en huertas caseras: el primero, basado en la utilización de un sustrato degradador inoculado con microorganismos de montaña, y el segundo, inoculado mediante 
el procedimiento Takakura. Ambos métodos presentaron indicadores de degradación de los residuos sólidos, con temperaturas superiores a $50^{\circ} \mathrm{C}$, una evolución de $\mathrm{pH}$ adecuada para este tipo de compostaje y una reducción en altura del volumen. Señalar que en el trabajo fueron inoculados microorganismos con la finalidad de acelerar el proceso de descomposición.
Durante el compostaje ocurren cambios cualitativos y cuantitativos en la microflora activa. Algunas especies se multiplican rápidamente al inicio cambiando el medio ambiente y luego desaparecen para permitir ser sucedidos por otras poblaciones de microorganismos.

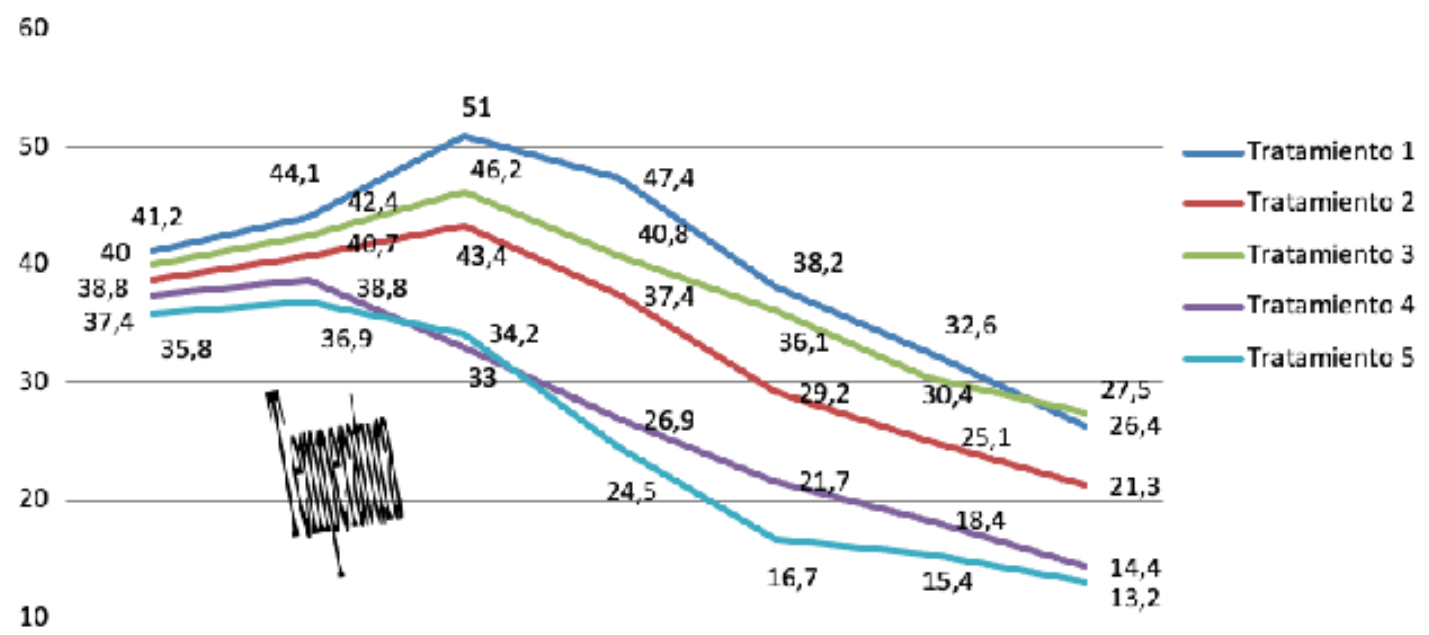

10

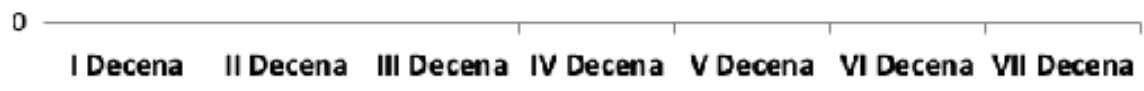

Figura 1. Comportamiento de la temperatura durante el compostaje.

\section{Descomposición del compost}

La Figura 2 refleja el porcentaje de descomposición en cada una de las variantes de compost evaluadas. El tratamiento 3: Caña de azúcar (Saccharum officinarum L.) + Estiércol vacuno $+5 \%$ de Leucaena leucocephala L. vc: Perú + Inoculante alcanzó el mayor porcentaje de descomposición $(88,6 \%)$, seguido del tratamiento 2 : King grass (Pennisetum purpureum Schumach vc: Común) + Estiércol vacuno $+5 \%$ de Leucaena leucocephala L. vc: Perú + Inoculante, con un $84,6 \%$, y el tratamiento 1: Caña de azúcar (Saccharum officinarum L.) + Estiércol vacuno + Inoculante con un $78,2 \%$, todos con diferencias estadísticas entre sí. El tratamiento 5: Paja de arroz (Oryza sativa L.) + Estiércol vacuno+Jacinto de agua (Eichhornia azurea K.S) + Inoculante mostró un 69,4\% de descomposición, mientras que el tratamiento 4: Jacinto de agua (Eichhornia azurea K.S) + Estiércol vacuno + 5\% de Leucaena leucocephala L. vc: Perú + Inoculante resultó el más bajo con un $68 \%$ de descomposición, ambo no difirieron estadísticamente.

Resulta evidente, que los tratamientos con mayor descomposición incluyeran en sus componentes iniciales residuos con una relación C/N alta (caña de azúcar: 64,84 y king grass: $62,23 \%$ respectivamente), sin embargo la adición de estiércol vacuno a los 
tres tratamientos y de Leucaena al segundo y tercero (sustratos con una relación $\mathrm{C} / \mathrm{N}$ de 31,10 y 16,26 respectivamente), pudo facilitar una mejor descomposición. Los tratamientos con menor descomposición también incluían en ambos casos al estiércol vacuno, mientras que incorporaban al Jacinto de agua (Eichhornia azurea K.S) y el tratamiento 5 descartó la leguminosa Leucaena leucocephala.

Se debe tener en cuenta que el compostaje resulta de un proceso biológico aerobio exotérmico ejecutado por microorganismos (bacterias y hongos principalmente) que descomponen biológicamente y mineralizan un sustrato orgánico, según la temperatura y otras condiciones de la composta (Escudero y Arias, 2012), por lo que en la descomposición alcanzada en los tratamientos 4 y 5, pudo influir el poco peso del Jacinto de agua que pudo "ser aprisionado" y provocar una compactación de la pila, disminuir la temperatura y enlentecer el proceso resultando en una descomposición más baja como se aprecia en la Figura 2.
El compost se obtuvo entre los 105-110 días aproximadamente en los tratamientos 1, 2 y 3, mientras que en el 4 y 5 se extendieron hasta los 135-140 días, y aunque tardaron más días para completar el proceso, esto no influyó en que presentaran mayor porcentaje de descomposición. Este comportamiento estuvo muy relacionado con la evolución de la temperatura en las pilas, el material empleado y las diferentes combinaciones. De forma visual se pudo apreciar los cambios que se produjeron en los residuos hasta su transformación en compost, si bien, al inicio se distinguía su coloración entre los restos frescos, paulatinamente se volvieron más oscuro, con una textura suave a la palpación.

Las características finales del compost estarán determinadas por el tipo de material que se aporte, la tecnología y duración del proceso. Resulta de buena calidad si el producto final es relativamente homogéneo, de color oscuro y olor a tierra de bosque (Soliva, 2011).

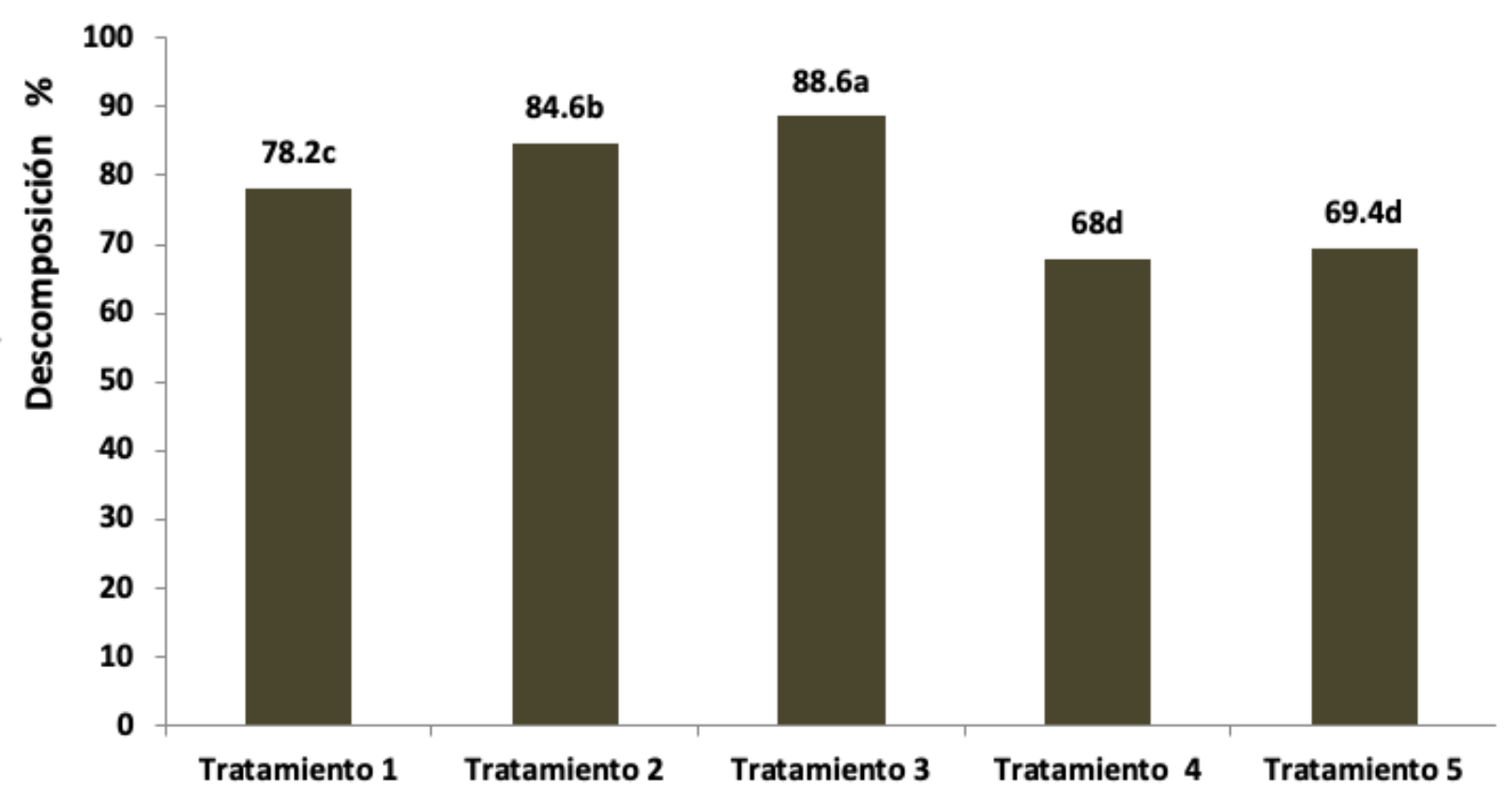

Figura 2. Porcentaje de descomposición del compost obtenido. Letras distintas indican diferencias significativas según la prueba de Tukey $(p \leq 0,05)$. 


\section{Caracterización del compost obtenido}

En la Tabla 3 se muestran algunas características químicas del compost obtenido, donde el mayor porcentaje de nitrógeno se encontró en el tratamiento 3 (Caña de azúcar (Saccharum officinarum L.) + Estiércol vacuno $+5 \%$ de Leucaena leucocephala L. vc: Perú + Inoculante, con 1,55\% que difirió estadísticamente del resto, mientras que los tratamientos 2 (King grass (Pennisetum purpureum Schumach vc: Común) + Estiércol vacuno $+5 \%$ de Leucaena leucocephala L. vc: Perú + Inoculante), tratamiento 4 (Jacinto de agua (Eichhornia azurea K.S) + Estiércol vacuno $+5 \%$ de Leucaena leucocephala L. vc: Perú + Inoculante) y tratamiento 1 (Caña de azúcar (Saccharum officinarum L.) + Estiércol vacuno + Inoculante) con $1,46 \%, 1,45 \%$ y $1,40 \%$ respectivamente no difirieron entre sí, este último sin diferencias del tratamiento 5 (Paja de arroz (Oryza sativa L.) + Estiércol vacuno + Jacinto de agua (Eichhornia azurea K.S) + Inoculante) que reportó el contenido de nitrógeno más bajo (1,31\%).

En cuanto al fósforo, no hubo diferencias entre los tratamientos y se mantuvo en un rango de $0,44-0,53 \%$. El potasio fue más alto en el tratamiento $3(1,87 \%)$, con diferencias del resto, seguido de los tratamientos $4(1,71 \%)$ y $2(1,69 \%)$, que no difirieron entre sí, mientras los valores más bajos fueron en los tratamientos
1 y 5 con 1,30\% y 0,97\% respectivamente. Martínez (2013), citado por Román etal., (2013), en un compost obtenido a partir de diferentes residuos orgánico reportaron contenido de nitrógeno $0,3 \%-1,5 \%$ (3 g a 15 g por $\mathrm{kg}$ de compost), fósforo $0,1 \%-1,0 \%$ (1g a $10 \mathrm{~g}$ por $\mathrm{kg}$ de compost) y potasio $0,3 \%-1,0 \%$ (3g a $10 \mathrm{~g}$ por kg de compost). Referido al nitrógeno y el fósforo, los resultados alcanzados en el trabajo se encuentran en ese rango, mientras el potasio fue más alto.

La materia orgánica fue superior en el tratamiento $3(62.80 \%)$, con diferencias del resto de los tratamientos, que no mostraron diferencias entre ellos, y ubicaron el contenido de materia orgánica entre 30,31\% y 35,29\%.

La relación $\mathrm{C} / \mathrm{N}$ en el tratamiento 3 resultó 23,29 y difirió de todos los tratamientos, seguida del tratamientos $5(14,73)$, que también fue estadísticamente diferente de los tratamientos restantes. Los tratamientos $2(13,89)$ y $4(13.79)$ no mostraron diferencias entre sí. El valor más bajo estuvo en el tratamiento 1(12,44). Resulta fundamental en el análisis de un material compostado la relación $\mathrm{C} / \mathrm{N}$, considerada por Rodríguez, et al., (2018), un indicador del origen, grado de madurez y estabilidad de la materia orgánica.

Tabla 3. Indicadores químicos del compost obtenido.

\begin{tabular}{|c|c|c|c|c|c|c|}
\hline Tratamientos & $\mathrm{N}(\%)$ & $\mathrm{P}(\%)$ & $\mathrm{K}(\%)$ & M.O (\%) & $\mathrm{C} / \mathrm{N}$ & $\mathrm{pH}$ \\
\hline 1 & $1,40^{\mathrm{bc}}$ & $0,44^{\mathrm{ns}}$ & $1,30^{c}$ & $30,31^{b}$ & $12,44^{\mathrm{d}}$ & $7,28^{\text {ns }}$ \\
\hline 2 & $1,46^{b}$ & $0,53^{\text {ns }}$ & $1,95^{\mathrm{a}}$ & $35,29^{b}$ & $13,89^{c}$ & $7,98^{\text {ns }}$ \\
\hline 3 & $1,55^{\mathrm{a}}$ & $0,53^{\mathrm{ns}}$ & $1,76^{b}$ & $62,80^{\mathrm{a}}$ & $23,29^{\mathrm{a}}$ & $7,87^{\mathrm{ns}}$ \\
\hline 4 & $1,45^{\mathrm{b}}$ & $0,44^{\text {ns }}$ & $1,71^{\mathrm{b}}$ & $34,80^{\mathrm{b}}$ & $13,79^{c}$ & $7,73^{\mathrm{ns}}$ \\
\hline 5 & $1,31^{\mathrm{c}}$ & $0,46^{\mathrm{ns}}$ & $0,97^{d}$ & $33,57^{b}$ & $14,73^{b}$ & $7,73^{\mathrm{ns}}$ \\
\hline es \pm & $0,136^{*}$ & $0,068^{\mathrm{ns}}$ & $0,207^{*}$ & $1,564^{*}$ & $0,094 *$ & $0,086^{\text {ns }}$ \\
\hline
\end{tabular}

Letras distintas indican diferencias significativas según la prueba de Tukey $(p \leq 0,05)$. 
De forma general, la relación $\mathrm{C} / \mathrm{N}$ estuvo entre 12,44 y 23,29/1; muy favorable resultó que no se obtuviera un compost mucho más rico en carbono, el que una vez aplicado al suelo como abono, podría propiciar un bloqueo biológico del nitrógeno, conocido como "hambre de nitrógeno", donde los microorganismos consumen el $\mathrm{C}$ presente en el material, $\mathrm{y}$ rápidamente incrementan la demanda de $\mathrm{N}$, agotando las reservas del mismo en el suelo (Román et al., 2013).

El Department of Sanitation, (2012), recomienda que la $\mathrm{C} / \mathrm{N}$ al final de un proceso de compostaje alcance un rango óptimo en valores cercanos a 20 y acota que la relación $\mathrm{C} / \mathrm{N}$ inicial en cada tratamiento es un factor determinante en la relación $\mathrm{C} / \mathrm{N}$ final del compost producido. En el $\mathrm{pH}$ final del compost, no hubo diferencias estadísticas entre los tratamientos, y el rango del mismo estuvo entre 7,28-7,98.

Respecto al pH del compostaje, (Mayea, 1992) planteó que el mismo dependerá de los materiales de origen y puede variar en cada fase del proceso (desde 4.5 a 8.5), encontrando que en los primeros estadios, el pH se acidifica por la formación de ácidos orgánicos. En la fase termófila, debido a la conversión del amonio en amoniaco, el pH sube y se alcaliniza el medio, para finalmente estabilizarse en valores cercanos al neutro cuando concluye el proceso de compostaje.

En relación a lo planteado por este autor, en muestras de observación realizadas en cada una de esta fases a los 25, 45, 100 y 125 días del proceso se obtuvieron valores promedio entre $4,63,5.6,7,70$ y 8,0 respectivamente (datos no mostrados), lo que pudiera coincidir con el comportamiento del $\mathrm{pH}$ citado anteriormente por el autor de referencia.

En el análisis del compost obtenido hay que tener en cuenta el material de origen y la evolución del proceso de compostaje, lo que coincide con D'Haene et al., (2014), cuando refieren que los intervalos óptimos de cada uno de los indicadores agroquímicos evaluados en un compost están influenciados por las condiciones ambientales, el tipo de residuo a tratar y el sistema de compostaje elegido.

\section{CONCLUSIONES}

El comportamiento de la temperatura mantuvo indistintamente en todos los tratamientos la secuencia de fases: Mesófila, Termófila y Mesófila II, con una duración del proceso de compostaje entre 105-110 días y un porcentaje de descomposición de 78,2\%, 84,6\% y $88,6 \%$ respectivamente en los tratamientos con Sacharum officinarum y Pennisetum purpureum.

En los tratamientos con Eichhornia azurea y paja de Oryza sativa el compostaje se extendió hasta los 135-140 días, con una descomposición de $68 \%$ y $69,4 \%$ respectivamente, sin que la extensión del proceso influyera en la misma.

El compost obtenido en los diferentes tratamientos resulta viable, desde el punto de vista de los indicadores técnicos evaluados, con destaque para el tratamiento 3: Caña de azúcar (Saccharum officinarum L.) + Estiércol vacuno $+5 \%$ de Leucaena leucocephala L. vc: Perú + Inoculante que mostró los mayores valores de nitrógeno $(1,55 \%)$, potasio $(1,87 \%)$ y materia orgánica $(62,8 \%)$, con un contenido de fósforo de $0,53 \%$, relación $\mathrm{C} / \mathrm{N}$ de $23,29, \mathrm{pH}(7,87)$ y la mayor descomposición.

\section{Conflicto de Intereses}

Los autores declaran que es un trabajo original y no existió conflicto de intereses de ningún tipo en la elaboración y publicación del manuscrito. 


\section{REFERENCIAS}

Alvarado, J., Andrade, H. J. y Segura, M. 2013. Almacenamiento de carbono orgánico en suelos en sistemas de producción de café (Coffea arabica L.) en el municipio del Líbano, Tolima, Colombia. Col. For. $16: 21-31$

Bohórquez, A., Puentes, Y, J. y Menjivar, J. C. 2014. Evaluación de la calidad del compost producido a partir de subproductos agroindustriales de caña de azúcar. Corpoica Cienc. Tecnol. Agropecu. 15(1): 73-81.

Campos, R., Brenes, L. y Jiménez, M. 2016. Evaluación técnica de dos métodos de compostaje para el tratamiento de residuos sólidos biodegradables domiciliarios y su uso en huertas caseras. Tecnología en Marcha. Encuentro de Investigación y Extensión. 25-32.

Doi: 10.18845/tm.v29i8.2982.

Carlile, W. R., Cattivello, C. and Zaccheo, P. 2015. Organic growing media: Constituents and properties. Vadose Zone Journal 14(6): 0125.

Casimiro, L. 2016. Necesidad de una transición agroecológica en Cuba, perspectivas y retos. Pastos y Forrajes, 39(3): 81-91.

D'Haene, K., Salomez, J., De Neve, S., De Waele, J. and Hofman. G. 2014. Environmental performance of nitrogen fertilizer limits imposed by the EU Nitrates Directive. Agriculture, Ecosystems \& Environment, 192:67-79.

Donn, S., Wheatley, R.E., Mckenzie, B.M., Loades, K.W. and Hallett, P.D. 2014. Improved soil fertility from compost amendment increases root growth and reinforcement of surface soil on slopes. Ecological Engineering, 71(0): 458-465.
Escudero, A. y Arias, C.A. 2012. Los microorganismos en los abonos orgánicos a partir de podas en la Universidad del Norte, Colombia. Rev. Int. Contam. Ambie. 28 Sup. (1) 67-75.

García, D. I., Lima, L.A., Ruíz, L. y Calderón, P.A. 2014. Métodos y parámetros para determinar la madurez en el compost a nivel de fincas. Cub@: Medio Ambiente y Desarrollo; Revista electrónica de la Agencia de Medio Ambiente. (26) ISSN-1683-8904.

Hernández, A., Pérez, J. M., Bosch, D. y Castro, N. 2015. Clasificación de los suelos de Cuba. Mayabeque, Cuba: Ediciones INCA, Instituto de Suelos.

López, E., Andrade, A.J., Herrera, M., Gonzalez, O. y García, A. 2017. Propiedades de un compost obtenido a partir de residuos de la producción de azúcar de caña, Revista Centro Agrícola. 44(3): 49-55.

Mayea, S. 1992. Tecnología para la producción de compost (biotierra) a partir de la inoculación con microorganismos de diversos restos vegetales. CIDA. MINAGRI. La Habana. 22 pp.

NC-ISO-10390. 1999a. Determinación del pH. La Habana: Oficina Nacional de Normalización. La Habana, Cuba.

NC-ISO-51. 1999b. Determinación de materia orgánica en suelo, La Habana: Oficina Nacional de Normalización.

NRAG-564. 2003. Tejido vegetal. Análisis Foliar. Métodos de ensayo. La Habana, Ministerio de la Agricultura

Departament of Sanitation New York City (NYC). 2012. Master composter. Manual. Department of Sanitation. NY. 158. 
Román, P., Martínez, M. y Pantoja, A. 2013. Manual de Compostaje del Agricultor Experiencias en América Latina. Organización de las Naciones Unidas para la Alimentación y la Agricultura, Oficina Regional para América Latina y el Caribe Santiago de Chile.112.

Rodríguez, S. G., Gómez, L. B., Couoh, E. V., Botello, M. A. E. y Garruña, R. 2018. Caracterización física y química de materiales orgánicos para sustratos agrícolas. Agrociencia .639-652.
Soliva, M. 2011. Materia orgánica y compostaje: control de la calidad y del proceso. Escuela Superior de Agricultura de Barcelona.

http://biomusa.net/es/jornadas-yactividades/jornada-tecnica-sobrecalidad-y-fertilidad-del-suelo/70materia-organica-y-compostaje-controlde-lacalidad/file

Vento, M., Pérez, Guevara, A., Corrales, I., Chaveli, P., Casañola, A., Valenciano, M. y Velasco, M. 2010. Compost a partir de residuos de cosechas y alternativas que mejoran su calidad. Centro Agrícola, 37(2):75-80. 